\title{
Cyclotron-resonance-assisted photon drag effect in InSb/InAISb quantum wells excited by terahertz radiation
}

\author{
S. Stachel, ${ }^{1}$ G. V. Budkin, ${ }^{2}$ U. Hagner, ${ }^{1}$ V. V. Bel'kov, ${ }^{2}$ M. M. Glazov, ${ }^{2}$ S. A. Tarasenko, ${ }^{2}$ S. K. Clowes, ${ }^{3}$ T. Ashley, ${ }^{4}$ \\ A. M. Gilbertson, ${ }^{5}$ and S. D. Ganichev ${ }^{1}$ \\ ${ }^{1}$ Terahertz Center, University of Regensburg, 93040 Regensburg, Germany \\ ${ }^{2}$ Ioffe Physical-Technical Institute, Russian Academy of Sciences, 194021 St. Petersburg, Russia \\ ${ }^{3}$ Advanced Technology Institute and SEPNet, University of Surrey, Surrey GU2 7XH, United Kingdom \\ ${ }^{4}$ School of Engineering, University of Warwick, Coventry CV4 7AL, United Kingdom \\ ${ }^{5}$ Blackett Laboratory, Imperial College, London SW7 2BZ, United Kingdom
}

(Received 13 January 2014; published 27 March 2014)

\begin{abstract}
We report on the observation of the cyclotron-resonance-assisted photon drag effect. Resonant photocurrent is detected in InSb/InAlSb quantum well structures subject to a static magnetic field and excited by terahertz radiation at oblique incidence. The developed theory based on Boltzmann's kinetic equation is in good agreement with the experimental findings. We show that the resonant photocurrent originates from the transfer of photon momentum to free electrons drastically enhanced at the cyclotron resonance.
\end{abstract}

DOI: 10.1103/PhysRevB.89.115435 PACS number(s): 72.40.+w, 73.21.Fg, 72.25.Fe, 72.25.Rb

\section{INTRODUCTION}

Indium antimonide based quantum well $(\mathrm{QW})$ structures exhibiting the highest room temperature electron mobility of any semiconductors are considered as prospective material for the next generation of electronic devices being of interest for such applications as high mobility transistors [1], ballistic transport based electronics [2-4], and spintronics [5-7]. The high mobility caused by the small effective electron mass together with large dielectric constant and narrow energy gap also make these nanostructures a prospective for midinfrared and terahertz optoelectronic applications [8-10]. Moreover, the photocurrent measurements were shown to provide a method for InSb-based QWs characterization giving an access to, e.g., the fine structure of electron energy spectrum and electron-electron exchange interaction [11,12].

Here we report on the observation and study of the photon drag effect induced by terahertz laser radiation in InSb/AlInSb QWs. The photocurrent is generated owing to the momentum transfer from photons to an electron ensemble: The phenomenon known for other materials and being of importance for material science as well as for ultrafast detection of IR/THz laser radiation [8-10]. We also show that this process is drastically enhanced under the cyclotron resonance (CR) conditions, leading to an improved performance of the photocurrent generation. An increase of the photon drag current magnitude by more than 50 times as compared to the photocurrent excited at zero field is detected at the magnetic field of about 1 to $3 \mathrm{~T}$ depending on the radiation frequency lying between 1 and $3.3 \mathrm{THz}$. A rather low magnetic field at which the $\mathrm{CR}$ resonance is detected results from the small electron effective mass [13-16]. We develop the quasiclassical theory of the cyclotron-resonance-assisted photon drag effect, which is in quantitative agreement with experimental data. Our results reveal that the photocurrent can be used as a complementary method to study electron scattering, effective mass, and energy dependence of the momentum relaxation time in InSb-based QW, being of particular importance at high temperatures, where transmission measurements are inefficient.

\section{SAMPLES AND EXPERIMENTAL TECHNIQUES}

The experiments are carried out on $30 \mathrm{~nm}$ wide $\mathrm{InSb}$ QW structures grown on (001)-oriented semi-insulting GaAs substrates by molecular beam epitaxy. The lower barrier of the well consists of $\operatorname{In}_{0.9} \mathrm{Al}_{0.1} \mathrm{Sb}$, while the top barrier is $\mathrm{In}_{0.85} \mathrm{Al}_{0.15} \mathrm{Sb}$ containing a Te $\delta$-doping layer. The $\mathrm{QW}$ hosts a two-dimensional electron gas (2DEG) with a carrier density of $n_{s}=5 \times 10^{11} \mathrm{~cm}^{-2}$ and a mobility of $\mu=1 \times 10^{5} \mathrm{~cm}^{2} / \mathrm{V} \mathrm{s}$ at $77 \mathrm{~K}$. The samples are square shaped with a length of $5 \mathrm{~mm}$. The sample edges are oriented along the $x \|[1 \overline{1} 0]$ and $y \|[110]$ crystallographic axes. Four ohmic contacts fabricated at the middle of each sample edge allow us to probe the photocurrent in $x$ and $y$ directions (see the inset in Fig. 1).

To generate photocurrents in unbiased samples we applied $\mathrm{cw}$ or pulsed terahertz $(\mathrm{THz})$ laser systems. As a source of $\mathrm{cw}$ radiation, a $\mathrm{CH}_{3} \mathrm{OH}$ laser operating at the frequency $2.54 \mathrm{THz}$ (photon energies $\hbar \omega=10.4 \mathrm{meV}$ ) was used. The incident power $P \approx 0.5 \mathrm{~mW}$ at the sample was modulated at $800 \mathrm{~Hz}$ by an optical chopper. The radiation at the oblique incidence was focused in a spot of about $1.5 \mathrm{~mm}$ diameter at the center of sample. The spatial beam distribution had an almost Gaussian profile which was measured by a pyroelectric camera [17]. The electric field $\boldsymbol{E}$ of linearly polarized laser radiation was oriented either parallel ( $p$ polarization) or perpendicular $(s$ polarization) to the plane of incidence. Note that in the latter case, the electric field vector laid in the QW plane. For the pulsed radiation, an $\mathrm{NH}_{3}$ laser $[9,18,19]$ operating at the frequencies $f=1.07,2.03$, and $3.31 \mathrm{THz}(\hbar \omega=4.4,8.4$, and $13.7 \mathrm{meV}$, respectively) was applied. The laser generates single pulses with a duration of about $100 \mathrm{~ns}$, peak power of $P \approx 5 \mathrm{~kW}$, and a repetition rate of $1 \mathrm{~Hz}$. The radiation power was controlled by the THz photon drag detector [20].

The geometry of the experiments is sketched in the insets in Figs. 1 and 2. Samples were excited by the laser beam lying in the $(x z)$ plane at the angle of incidence $\theta_{0}$, varied between $-30^{\circ}$ and $+30^{\circ}$, with respect to the layer normal $z \|$ [001]. Both the current components perpendicular $\left(j_{y}\right)$ and parallel $\left(j_{x}\right)$ to the plane of incidence were investigated. The corresponding photocurrents were measured by the voltage 


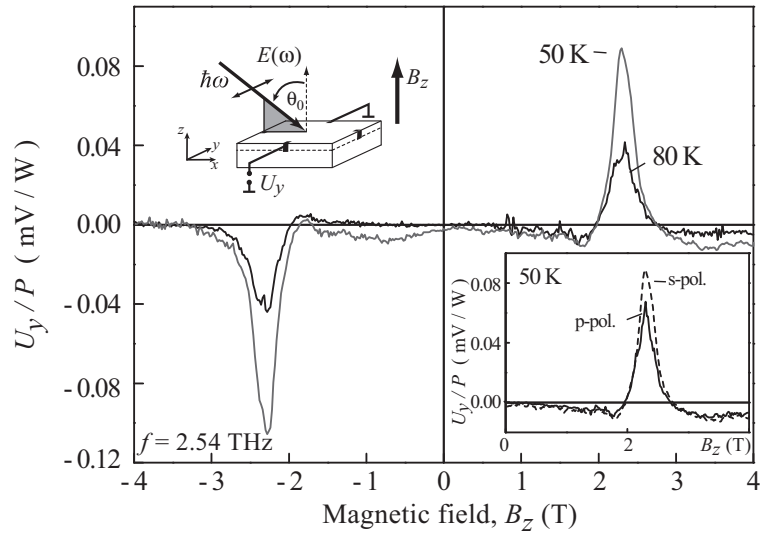

FIG. 1. Photosignal $U_{y}$ normalized by the radiation power $P$ excited by linearly polarized light ( $s$ polarization) with $f=2.54 \mathrm{THz}$ as a function of the magnetic field $B_{z}$, incident angle $\theta_{0}=20^{\circ}$. The left inset shows the experimental geometry. In the right one the field dependencies for $s$ - and $p$-polarized radiation are presented.

drops $U_{x(y)}$ picked up across a $1 \mathrm{M} \Omega$ (cw measurements) or $50 \Omega$ (pulsed measurements) load resistors. To record the signal in the former and the latter cases, a lock-in amplifier and digital oscilloscope were applied, respectively. Complimentary transmission measurements were performed applying a cw laser operating at the frequencies $f=1.63$ or $2.54 \mathrm{THz}$ and the Golay cell as a sensitive radiation detector. These measurements were done at normal incidence of radiation using linearly polarized as well as right-handed $\left(\sigma^{+}\right)$and left-handed $\left(\sigma^{-}\right)$circularly polarized light obtained by $\lambda / 4$ plates. Samples were mounted in a temperature variable magneto-optical cryostat. Experiments were carried out in the temperature range between 4.2 and $120 \mathrm{~K}$ and the magnetic field up to $7 \mathrm{~T}$ applied along the $z$ direction.

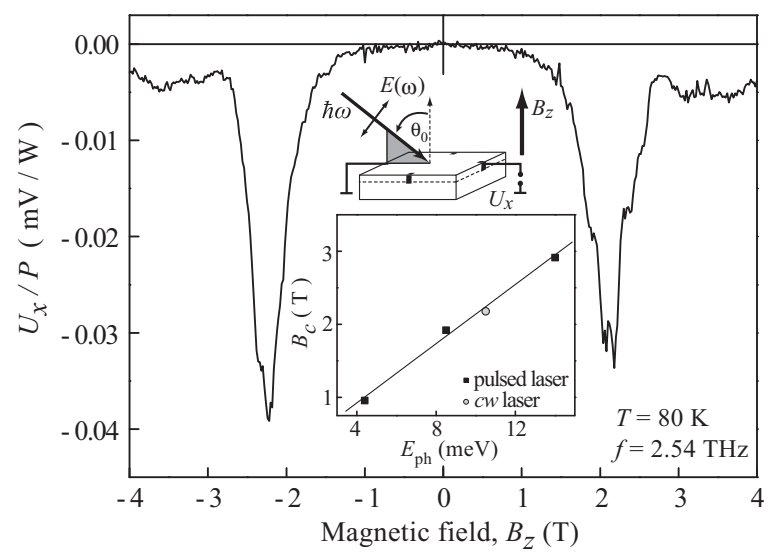

FIG. 2. Photosignal $U_{x}$ normalized by the radiation power $P$ excited by linearly polarized light ( $p$ polarization) with $f=2.54 \mathrm{THz}$ as a function of the magnetic field $B_{z}$, incident angle $\theta_{0}=20^{\circ}$. The top inset shows the experimental geometry. The bottom one demonstrates magnetic field positions $B_{c}$ of the resonant photosignals induced by the $\mathrm{cw}(f=2.54 \mathrm{THz})$ and pulsed laser systems as a function of the photon energy $E_{\mathrm{ph}}$ obtained at $T=4.2 \mathrm{~K}$.

\section{RESULTS}

We start with the data obtained at rather high temperatures (above $\approx 20 \mathrm{~K}$ ) and applying low power radiation of $\mathrm{cw}$ laser operating at $f=2.54 \mathrm{THz}$. Exciting the sample with linearly polarized radiation at oblique incidence [21] and sweeping magnetic field, we observed a resonant photosignal with the maximum at $\left|B_{c}\right|=2.3 \mathrm{~T}$, see Figs. 1 and 2. The resonant photoresponses are detected both in perpendicular and parallel to the light incident plane directions. However, the signal parities are different: In the former case $\left(U_{y}\right)$ it is odd (Fig. 1) while in the latter case $\left(U_{x}\right)$ it is even (Fig. 2) in the magnetic field $B_{z}$. As an important result, the magnitude of the resonances is almost independent of the orientation of the radiation electric field vector $\boldsymbol{E}_{\omega}$ with respect to the QW plane. The photocurrents excited by the radiation polarized in the QW plane ( $s$ polarization, $\boldsymbol{E}_{\omega} \| y$ ) and the field with the orthogonal polarization ( $p$ polarization, $E_{\omega, z} \neq 0$ ) are shown in the inset in Fig. 1.

In the temperature range between 20 and $100 \mathrm{~K}$, the resonances are well described by the Lorentz functions and the decrease of temperature only causes the increase of its magnitude. For lower temperatures, $T<20 \mathrm{~K}$, the magnetic field behavior of the photocurrent becomes more complicated. Figure 3 shows that now the photosignal oscillates upon variation of magnetic field. The amplitude of the oscillations increases with rising $B_{z}$ at low fields, achieves maximum at $B_{z} \approx B_{c}$, and decreases for higher magnetic fields. Analysis of the data reveals that the oscillations are periodic with $1 / B_{z}$. This is shown in Fig. 4 demonstrating a linear dependence of the inverse magnetic field positions $1 / B_{\max }$ of the photosignal maxima on an integer index $N$. Additional magnetotransport measurements demonstrate that the period of the observed oscillations is twice as that of the Shubnikov-de Haas oscillations ( $\mathrm{SdH}$ ), see Fig. 4. At substantially higher radiation intensities obtained applying pulsed terahertz laser, the oscillations vanish even at low sample temperatures, see inset in Fig. 3.

The magnetic field $B_{c}$ corresponding to the resonance versus the photon energy are shown in the inset in Fig. 2. Almost Lorentz shape of the resonance, linear scaling of $B_{c}$ with the radiation frequency, and the magnitudes of $B_{c}$ indicate that the resonances are caused by the cyclotron absorption of radiation. This conclusion is supported by the magnetic field dependence of the radiation transmission measured for normally incident radiation of the $\mathrm{cw}$ laser. Figure 5 shows that the data obtained for linearly and circularly polarized radiation with $f=2.54 \mathrm{THz}$ have a characteristic cyclotron resonance behavior: While for left-handed (right-handed) polarized light the transmission shows a sharp decrease at $B_{c} \approx-2.3 \mathrm{~T}(+2.3 \mathrm{~T})$ only for one magnetic field polarity, for linear polarization the dips in the transmission are observed for both magnetic field polarities and the dip magnitudes are reduced by the factor of 2 . Additional measurements applying cw laser radiation with $f=1.63 \mathrm{THz}$ prove that the resonance position shifts linearly with the radiation frequency as it is expected for the cyclotron resonance, see the inset in Fig. 5. The effective mass calculated from the $\mathrm{CR}$ is $m^{*}=0.023 \cdot m_{0}$ being in good agreement with the literature data on similar structures $[14,15,24]$. The difference between the cyclotron mass determined by the in-plane dispersion 


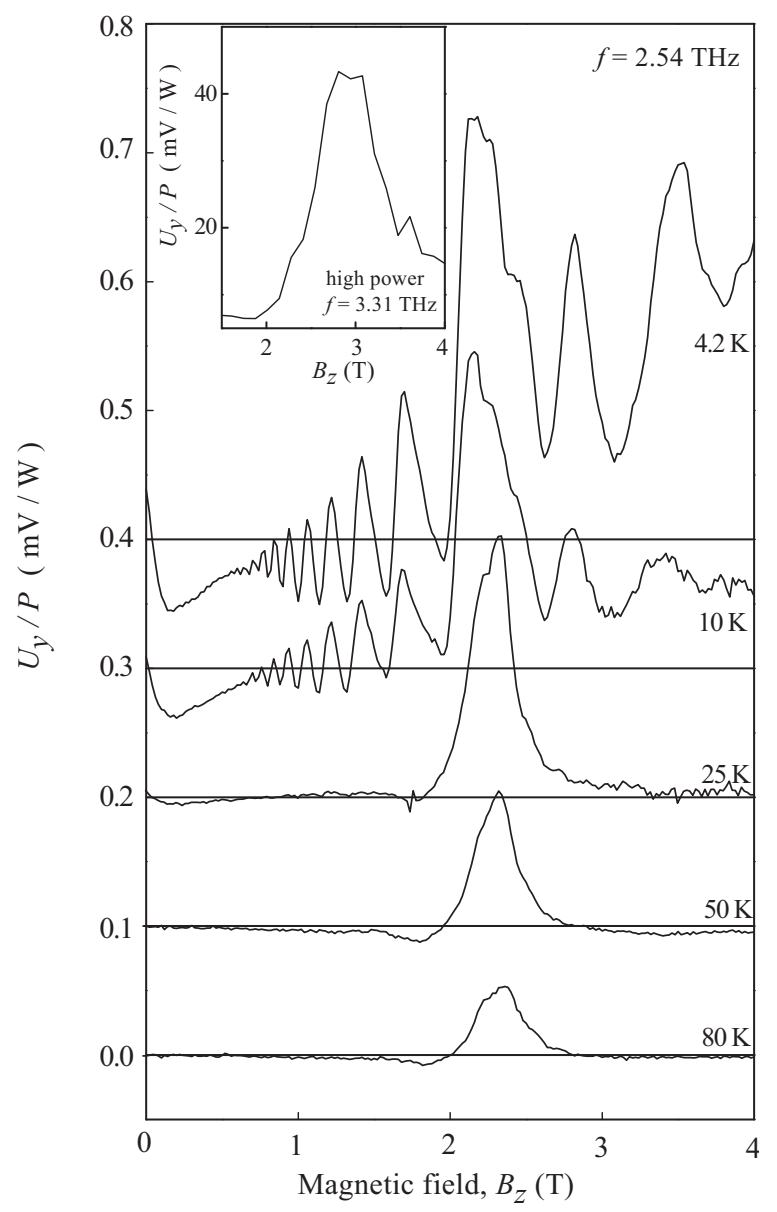

FIG. 3. Magnetic field dependence of $U_{y} / P$ for linearly polarized radiation of $f=2.54 \mathrm{THz}$ and different temperatures $T$. The data for $T<80 \mathrm{~K}$ are consistently shifted by $+0.1 \mathrm{mV} / \mathrm{W}$ for each step in temperature. Inset shows photovoltage resonance observed at $4.2 \mathrm{~K}$ under high power excitation of the pulse laser, $f=3.31 \mathrm{THz}$.

in the size-quantized subband and the effective mass at the conduction-band bottom of bulk InSb, $m_{\mathrm{eff}} \approx 0.014 \cdot m_{0}$, is caused by spectrum nonparabolicity in the bulk material. Momentum relaxation time determined from the resonance width $\left(1.1 \times 10^{-12} \mathrm{~s}\right)$ is found to be close to that obtained from the transport measurement also carried out at $4.2 \mathrm{~K}$ and yielding $\tau_{p}=1.3 \times 10^{-12} \mathrm{~s}$.

\section{SYMMETRY ANALYSIS}

Photocurrents induced by homogeneous excitation of QW structures with terahertz radiation can generally be caused by photogalvanic effects (PGE), resulting from an asymmetry of the carrier photoexcitation/scattering in $\boldsymbol{k}$ space [25], or photon drag effects (PDE), originating from the transfer of the photon momentum $\boldsymbol{q}$ to free carriers [9]. We start with the symmetry analysis of both effects and demonstrate that the observation of the photocurrent for $s$-polarized radiation allows us to exclude PGE as a possible origin of the photocurrent and, consequently, indicates that the current is caused by the photon drag effect. PGE and PDE are phenomenologically described by an equation which couples dc electric current with the

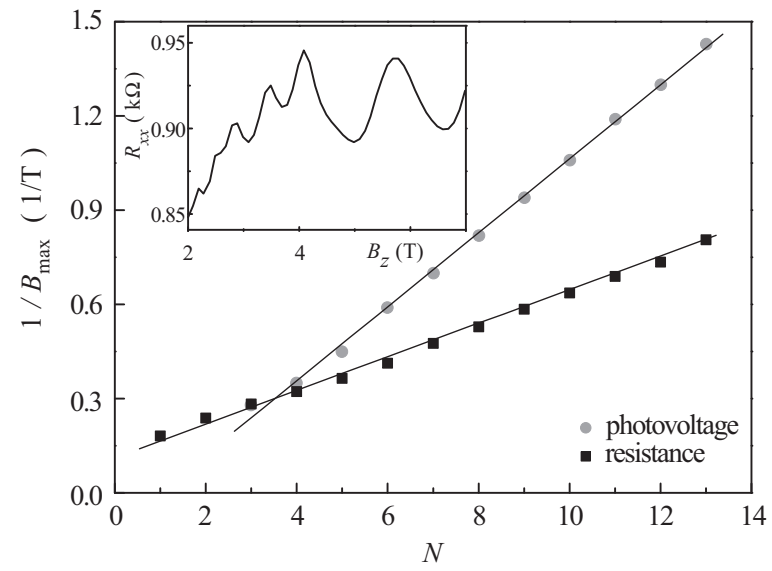

FIG. 4. Oscillation maxima of the photocurrent and SdH oscillations measured at $T=4.2 \mathrm{~K}$ are assigned an integer index $N$. Their $1 / B$-field positions are plotted versus the index. The inset shows the longitudinal resistance $R_{x x}\left(B_{z}\right)$ measured in the van der Pauw geometry for a constant current of $1 \mu \mathrm{A}$. Note that the $\mathrm{SdH}$ oscillations are superimposed by a high background signal resulting from the parallel conductance of the $\delta$-doping layer in the AlInSb barrier which is well known for InSb/AlInSb QWs [22,23].

amplitude and wave vector of the electromagnetic field inside the medium. To second order in the field amplitude (linear regime in the radiation intensity) and first order in the wave vector, such equation has the form $[9,26]$

$$
j_{\alpha}=\sum_{\beta \gamma} \chi_{\alpha \beta \gamma}(B) E_{\omega, \beta} E_{\omega, \gamma}^{*}+\sum_{\beta \gamma \delta} \phi_{\alpha \beta \gamma \delta}(B) q_{\beta} E_{\omega, \gamma} E_{\omega, \delta}^{*},
$$

where $\boldsymbol{j}$ is current density, $\boldsymbol{E}_{\omega}$ is the complex amplitude of the electric field of $\mathrm{THz}$ wave

$$
\boldsymbol{E}(\boldsymbol{r}, t)=\boldsymbol{E}_{\omega} e^{i \boldsymbol{q} \cdot \boldsymbol{r}-i \omega t}+\boldsymbol{E}_{\omega}^{*} e^{-i \boldsymbol{q} \cdot \boldsymbol{r}+i \omega t},
$$

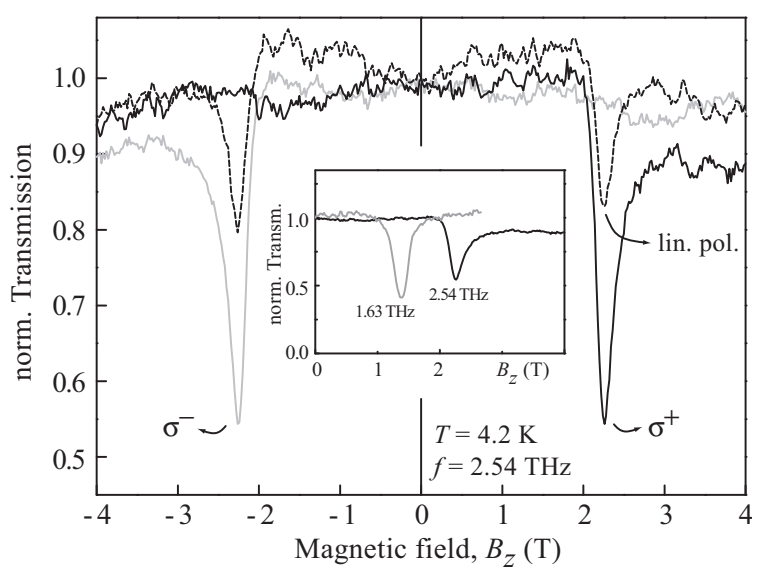

FIG. 5. Magnetic field dependence of the normalized transmission for right and left circularly as well as linearly polarized radiation at normal incidence of light. The transmitted radiation power is normalized by that at zero magnetic field. The data are obtained for $T=4.2 \mathrm{~K}$. The inset shows transmission curves for two radiation frequencies of the $\mathrm{cw}$ laser. 
$\boldsymbol{q}$ is the photon wave vector, and the indices $\alpha, \beta, \gamma, \delta$ enumerate the Cartesian components. The tensors $\chi$ and $\phi$ describe the photogalvanic effects and photon drag effects, respectively, and may depend on static magnetic field $\boldsymbol{B}$.

Our experiments are carried out on (001)-grown quantum wells which are described by the $C_{2 v}$ point group. For these structures subjected to the magnetic field $\boldsymbol{B}$ parallel to the growth direction $z$, the first term in the right-hand side of Eq. (1) is given by [27]

$$
\begin{aligned}
& j_{x}^{\mathrm{PGE}}=\chi_{x x z}\left(B_{z}\right) E_{\omega, x} E_{\omega, z}^{*}+\chi_{x y z}\left(B_{z}\right) E_{\omega, y} E_{\omega, z}^{*}+\text { c.c., } \\
& j_{y}^{\mathrm{PGE}}=\chi_{y y z}\left(B_{z}\right) E_{\omega, y} E_{\omega, z}^{*}+\chi_{y x z}\left(B_{z}\right) E_{\omega, x} E_{\omega, z}^{*}+\text { c.c. },
\end{aligned}
$$

where $\chi_{x x z}\left(B_{z}\right)$ and $\chi_{y y z}\left(B_{z}\right)$ are even functions of the magnetic field, $\chi_{x y z}\left(B_{z}\right)$ and $\chi_{y x z}\left(B_{z}\right)$ are odd functions, and $x \|[1 \overline{10}]$ and $y \|[110]$ are the in-plane axes. Equations (3) show that all contributions of the photogalvanic effect require a nonzero component of the radiation electric field along the growth direction $E_{\omega, z}$. Thus, the observation of the substantial photocurrent excited by $s$-polarized light $\left(E_{\omega, z}=0\right)$, rules out the photogalvanic effect as an origin of the observed resonant photocurrent (see inset in Fig. 1).

Now we turn to the photon drag effect described by the second term in the right-hand side of Eq. (1). Symmetry analysis shows that, in contrast to photogalvanics, photon drag effect is allowed for both $s$ and $p$ polarizations. In the case of $s$-polarized radiation and the $(x z)$ incident plane, i.e., for the $\boldsymbol{E}_{\omega} \| y$, the current density is given by

$$
\begin{aligned}
& j_{x}^{\mathrm{PDE}}=\phi_{x x y y}\left(B_{z}\right) q_{x}\left|E_{\omega, y}\right|^{2}, \\
& j_{y}^{\mathrm{PDE}}=\phi_{y x y y}\left(B_{z}\right) q_{x}\left|E_{\omega, y}\right|^{2},
\end{aligned}
$$

where $\phi_{x x y y}\left(B_{z}\right)$ is an even function of the magnetic field and $\phi_{y x y y}\left(B_{z}\right)$ is an odd function. Consequently, we obtain that the longitudinal photocurrent $\left(j_{x}\right)$ is even in the magnetic field and the transverse one $\left(j_{y}\right)$ is odd. Similar results are obtained for $p$-polarized radiation.

Polarization dependence and different parity of magnetic field dependencies of longitudinal and transverse photocurrent components, together with the negligible contribution of the photogalvanic effect, suggest that the photocurrent is dominated by the photon drag effect whose microscopic theory is considered in the next section.

\section{MICROSCOPIC THEORY}

We now turn to microscopic mechanisms responsible for photocurrent generation. For that we develop a quasiclassical theory of the cyclotron-resonance-assisted photon drag effect and show that a direct electric current emerges in the QW due to the action of electric and magnetic fields of the electromagnetic wave on charge carriers. Such a description is relevant to our experiments where highly doped $\mathrm{InSb} / \mathrm{InAlSb}$ quantum wells with the Fermi energy about $50 \mathrm{meV}$ are excited by terahertz radiation with at least by an order of magnitude smaller energy of photon. In this approach, the second order response is calculated in the framework of the Boltzmann kinetic equation for the electron distribution function $f(\boldsymbol{p}, \boldsymbol{r}, t)$ in the momentum, coordinate, and time spaces:

$$
\frac{\partial f}{\partial t}+\boldsymbol{v} \frac{\partial f}{\partial \boldsymbol{r}}+e\left(\boldsymbol{E}(\boldsymbol{r}, t)+\frac{1}{c}[\boldsymbol{v} \times \boldsymbol{B}(\boldsymbol{r}, t)]\right) \frac{\partial f}{\partial \boldsymbol{p}}=\mathrm{St}[f],
$$

where $\boldsymbol{v}=\boldsymbol{p} / m^{*}$ is the electron velocity, $e$ is the electron charge, $\boldsymbol{B}(\boldsymbol{r}, t)=\boldsymbol{B}+\boldsymbol{B}_{\omega} e^{i \boldsymbol{q} \cdot \boldsymbol{r}-i \omega t}+\boldsymbol{B}_{\omega}^{*} e^{-i \boldsymbol{q} \cdot \boldsymbol{r}+i \omega t}$ is the total magnetic field consisting of the static field $\boldsymbol{B} \| z$ and alternating magnetic field of the $\mathrm{THz}$ wave with the magnitude $B_{\omega}$, and $\mathrm{St}[f]$ is the collision integral.

The electric current density is given by the standard equation $\boldsymbol{j}=2 e \sum_{\boldsymbol{p}} \boldsymbol{v} f(\boldsymbol{p}, \boldsymbol{r}, t)$, where a factor of 2 accounts for spin degeneracy. The dc photoresponse is determined by the nonequilibrium corrections to the distribution function which are proportional to $E_{\omega, \|} B_{\omega, z}$ and $q_{\|} E_{\omega, \|}^{2}$, where the symbol " $\| "$ denotes the in-plane components. The terms yield two contributions to the current: (i) due to the dynamic Hall effect resulting from the joint action of electric and magnetic fields of the radiation [30] and (ii) due to spatial oscillations of the electric field and, consequently, electron distribution [31]. They were consistently considered for bulk materials [31] and two-dimensional systems with linear dispersion [32,33]. Note that the former contribution can be also rewritten via the photon wave vector giving $q_{\|} E_{\omega, \|}^{2}$, since the amplitudes of electric and magnetic fields in the plane wave are coupled by $\boldsymbol{B}_{\omega}=c\left[\boldsymbol{q} \times \boldsymbol{E}_{\omega}\right] / \omega$. Application of the static magnetic field $\boldsymbol{B} \| z$, besides deflecting the current due to the Hall effect, leads to the drastic enhancement of the photon drag effect at cyclotron resonance [34]. Estimations for the resonant photocurrent in InAs/GaSb structures is given in Ref. [35].

The details of calculations are as follows. We solve the kinetic equation Eq. (5) by expanding the distribution function $f(\boldsymbol{p}, \boldsymbol{r}, t)$ in the Fourier series of frequency, angular in the momentum space, and spatial harmonics

$$
f=\sum_{n, m, l} f^{n, m, l}(p) \exp \left(-i n \omega t+i m \varphi_{\boldsymbol{p}}+i l \boldsymbol{q}_{\|} \cdot \boldsymbol{r}\right),
$$

where $\varphi_{\boldsymbol{p}}=\arctan \left(p_{y} / p_{x}\right)$ is the polar angle of the vector $\boldsymbol{p}$. Note that in thermal equilibrium, the distribution function is described by the harmonic $f^{0,0,0}(p)=\left\{1+\exp \left[\left(\varepsilon_{p}-E_{F}\right) / k_{B} T\right]\right\}^{-1}$, with $\varepsilon_{p}=p^{2} /$ $\left(2 m^{*}\right)$ being the electron energy, $E_{F}$ is the Fermi energy, and $T$ is the temperature, while all other harmonics vanish. For elastic or quasielastic electron scattering by impurities or phonons, the collision integral takes the form

$$
\mathrm{St}[f]=-\sum_{n, l} \sum_{m \neq 0} \frac{f^{n, m, l}(p)}{\tau_{m}} \exp \left(-i n \omega t+i m \varphi_{\boldsymbol{p}}+i l \boldsymbol{q}_{\|} \cdot \boldsymbol{r}\right),
$$

where $\tau_{m}$ is the relaxation time of the $m$ th angular harmonic of distribution function. In the Fourier series representation, Eq. (5) has the form of linear equation system for the harmonics $f^{n, m, l}(p)$,

$$
\begin{gathered}
\Gamma^{n, m} f^{n, m, l}-i \frac{m e}{c m^{*}}\left(B_{\omega} f^{n-1, m, l-1}+B_{\omega}^{*} f^{n+1, m, l+1}\right) \\
+\frac{e \boldsymbol{E}_{\omega}}{2}\left(\boldsymbol{o}_{-} \hat{K}_{-}^{m} f^{n-1, m-1, l-1}+\boldsymbol{o}_{+} \hat{K}_{+}^{m} f^{n-1, m+1, l-1}\right)
\end{gathered}
$$




$$
\begin{aligned}
& +\frac{e \boldsymbol{E}_{\omega}^{*}}{2}\left(\boldsymbol{o}_{-} \hat{K}_{-}^{m} f^{n+1, m-1, l+1}+\boldsymbol{o}_{+} \hat{K}_{+}^{m} f^{n+1, m+1, l+1}\right) \\
& +i \frac{\operatorname{lv} \boldsymbol{q}_{\|}}{2}\left(\boldsymbol{o}_{-} f^{n, m-1, l}+\boldsymbol{o}_{+} f^{n, m+1, l}\right)=0
\end{aligned}
$$

where $\Gamma^{n, m}=1 / \tau_{m}-i n \omega-i m \omega_{c}, \omega_{c}=e B_{z} /\left(m^{*} c\right)$ is the cyclotron frequency, $\boldsymbol{o}_{ \pm}=\boldsymbol{o}_{x} \pm i \boldsymbol{o}_{y}, \boldsymbol{o}_{x}$ and $\boldsymbol{o}_{y}$ are the unit vectors along $x$ and $y$, respectively, and $\hat{K}_{ \pm}^{m}=\partial_{p} \pm(m \pm 1) / p$.
Straightforward calculations show that for $\boldsymbol{q}_{\|} \| x$ and degenerate electron gas the components of the drag current density have the form

$$
j_{x}^{\mathrm{pd}}=\operatorname{Re}(\tilde{j}), \quad j_{y}^{\mathrm{pd}}=-\operatorname{Im}(\tilde{j}),
$$

where

$$
\begin{aligned}
\tilde{j}= & \frac{q_{\|} e^{3}\left|E_{\omega, \|}\right|^{2} n_{s}}{\omega m^{* 2}} \frac{\tau_{p}}{1-i \omega_{c} \tau_{p}} \sum_{ \pm}\left\{\tau_{p} \frac{1 \pm \xi_{3}}{1+\left(\omega \pm \omega_{c}\right)^{2} \tau_{p}^{2}}+\frac{1}{2} \frac{\tau_{p}^{\prime} E_{F}}{1-i \omega_{c} \tau_{p}}\left[\left(\frac{\xi_{1}-i \xi_{2}}{1+i\left( \pm \omega-\omega_{c}\right) \tau_{p}}+\frac{1 \pm \xi_{3}}{1+i\left( \pm \omega+\omega_{c}\right) \tau_{p}}\right)\right.\right. \\
& \left.\left. \pm \frac{i \omega \tau_{2}\left(1 \mp \xi_{3}\right)}{\left[1+i\left( \pm \omega-2 \omega_{c}\right) \tau_{2}\right]\left[1+i\left( \pm \omega-\omega_{c}\right) \tau_{p}\right]}\right]\right\}
\end{aligned}
$$

$n_{s}=m^{*} E_{F} /\left(\pi \hbar^{2}\right)$ is the electron density, $\tau_{p} \equiv \tau_{1}$ is momentum relaxation time, $\tau_{p}^{\prime}=d \tau_{p}\left(E_{F}\right) / d E_{F}$, and parameters $\xi_{1}$, $\xi_{2}$, and $\xi_{3}$ are determined by polarization state of radiation and connected to the Stokes parameters [36]. The parameters are given by $\xi_{1}=\left(\left|E_{\omega, x}\right|^{2}-\left|E_{\omega, y}\right|^{2}\right) / E_{\omega, \|}^{2}, \quad \xi_{2}=\left(E_{\omega, x} E_{\omega, y}^{*}+\right.$ $\left.E_{\omega, x}^{*} E_{\omega, y}\right) / E_{\omega, \|}^{2}$, and $\xi_{3}=i\left(E_{\omega, x} E_{\omega, y}^{*}-E_{\omega, x}^{*} E_{\omega, y}\right) / E_{\omega, \|}^{2}$, the latter one reflecting the radiation helicity. Equation (10) shows that the photon drag current consists of two contributions: one being proportional to $\tau_{p}$ (first term in the curly brackets) and the other to the first derivative $\tau_{p}^{\prime}$. Remarkably that, while the first contribution exhibits the resonance at CR, i.e., for $\omega=\omega_{c}$, the second one has an additional resonance at the double frequency, $\omega=2 \omega_{c}$, where the radiation absorption does not have peculiarity. Dependence of the drag current on the polarization parameters $\xi_{1}$ and $\xi_{2}$ in two-dimensional systems with parabolic energy spectrum arises to the extent of energy dependence of the momentum relaxation time.

Finally, for the case of weak energy dependence of the momentum relaxation time, i.e., $\tau_{p}^{\prime} \ll \tau_{p} / E_{F}$, which is relevant for short-range scattering [37], and linearly polarized radiation, Eq. (10) reduces to

$$
\begin{aligned}
& j_{x}^{\mathrm{pd}}=\frac{q_{\|} e^{3}\left|E_{\omega, \|}\right|^{2} n_{s}}{\omega m^{* 2}} \frac{\tau_{p}^{2}}{1+\left(\omega_{c} \tau_{p}\right)^{2}} \sum_{ \pm} \frac{1}{1+\left(\omega \pm \omega_{c}\right)^{2} \tau_{p}^{2}}, \\
& j_{y}^{\mathrm{pd}}=-\omega_{c} \tau_{p} j_{x}^{\mathrm{pd}} .
\end{aligned}
$$

In the next section we analyze this result, calculate the dependence of photovoltage components on the magnetic field, and show that the theory describes well all experimental findings.

\section{DISCUSSION}

The above theory is developed in the assumption of a weak electron gas heating which is relevant to our experiments applying low power radiation of the $\mathrm{cw}$ laser. In these measurements the corresponding photocurrents have been detected by the voltage drops $U_{x, y}$ picked up across a 1 $\mathrm{M} \Omega$ load resistors being much larger than that of the $\mathrm{QW}$ structures. To obtain $U_{x, y}$ from Eq. (11) the static distribution of the electric potential $\Phi(r)$ have been found by solving the continuity equation $\operatorname{div} \boldsymbol{j}=0$ with boundary conditions setting the zero electric current across the sample edges (Fig. 6). Here the total electric current $j$ is written in the form

$$
j_{\alpha}=j_{\alpha}^{\mathrm{pd}}-\sum_{\beta=x, y} \sigma_{\alpha \beta} \nabla_{\beta} \Phi,
$$

where $j_{\alpha}^{\mathrm{pd}}$ is the local current density given by Eq. (11) and the second term describes the drift current with the components of the conductivity tensor in a static magnetic field $\sigma_{x x}=\sigma_{y y}=$ $\sigma_{0} /\left[1+\left(\omega_{c} \tau_{p}\right)^{2}\right], \sigma_{x y}=\sigma_{y x}=-\omega_{c} \tau_{p} \sigma_{0} /\left[1+\left(\omega_{c} \tau_{p}\right)^{2}\right]$, and the zero-field conductivity $\sigma_{0}=n e^{2} \tau_{p} / m^{*}$.

Figures 7 and 8 show dependencies of the photovoltages $U_{x, y}$ on magnetic field. The calculations are carried out using the momentum relaxation time $\tau_{p}=1.1 \times 10^{-12} \mathrm{~s}$ and effective mass $m^{*}=0.023 \cdot m_{0}$ obtained by the transmission measurements, the incident angle $\theta_{0}=20^{\circ}$, and taking into account the sample dimensions, the position of the contacts [38], and the Gaussian laser beam profile, see Fig. 6. Comparing these results with the corresponding experimental data shown in Figs. 1 and 2 demonstrate that all essential qualitative features of the observed photocurrent are well described by the microscopic model of the cyclotron-resonance-assisted photon drag effect. In particular, for linearly polarized radiation $U_{y}$ and

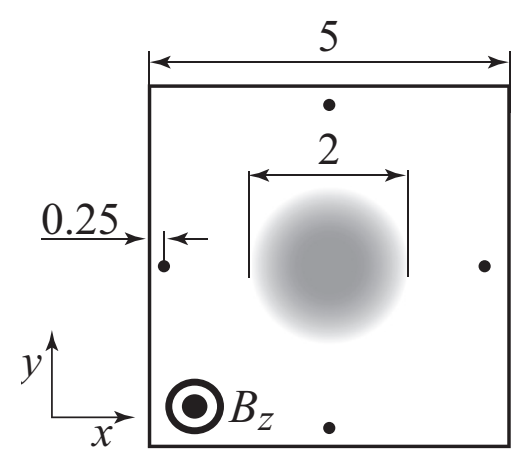

FIG. 6. Sample and contact positions, sizes are given in millimeters. Gray circle shows the laser spot located in the center of the sample. The spot has the Gaussian profile of about $2 \mathrm{~mm}$ diameter. Voltage drops $U_{x}$ and $U_{y}$ are measured between opposite contacts. 


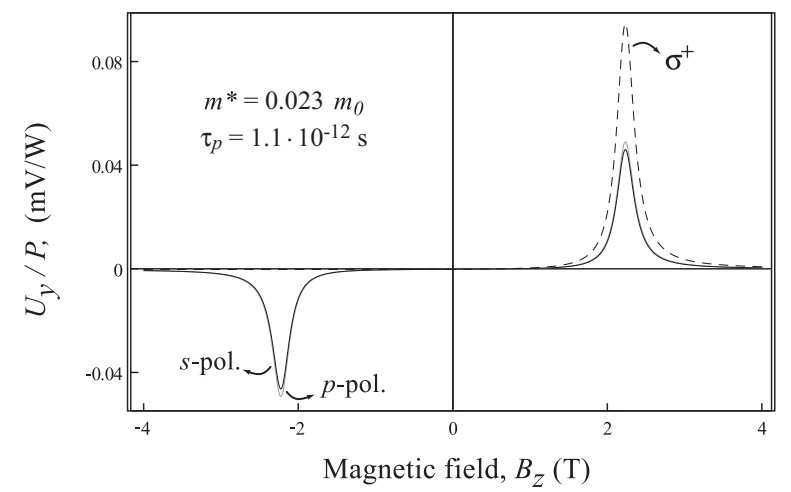

FIG. 7. Theoretical dependencies of $U_{y} / P$ on magnetic field for $s, p$, and $\sigma^{+}$polarizations. In the calculations the transmission coefficients $t_{s}$ and $t_{p}$ are set to unity for simplicity.

$U_{x}$ are described by the odd and even in magnetic field dependencies. Estimations of the photovoltage magnitude, carried out after Eqs. (11) and (12) and using parameters determined from the transport and CR transmission measurements, yield $U_{x}=0.05 \mathrm{mV} / \mathrm{W}$ for degenerate $2 \mathrm{DEG}$ which is in a good agreement with value obtained in the experiments. Both the theory and experiment show that the photocurrent at resonance comparing to the one excited at zero magnetic field is enhanced by 50 times (see, e.g., the data for $T=50 \mathrm{~K}$ in Fig. 1) or even more, see Figs. 1, 2, 7, and 8. Note that in another narrow band material, InAs/GaSb QWs, only a weak resonance with an amplitude comparable to that at zero magnetic field has been detected [35].

Alike CR transmission measurements fit of the photocurrent data by Eqs. (11) allows us to obtain the momentum relaxation times which found to be equal to $0.9 \times 10^{-12}$ and $0.7 \times 10^{-12} \mathrm{~s}$ for $T=50$ and $80 \mathrm{~K}$, respectively. The former value and the reduction of the relaxation time with the temperature increase are in a good agreement with the magnetotransport measurements. Moreover, our data provide even deeper insight in the scattering mechanisms. Indeed, the experimental data are well described by the calculations after Eqs. (11) and (12) which were obtained assuming a weak energy dependence of the momentum relaxation time, i.e., $\tau_{p}^{\prime} \ll \tau_{p} / E_{F}$. The validity of this assumption manifests itself in the magnetic

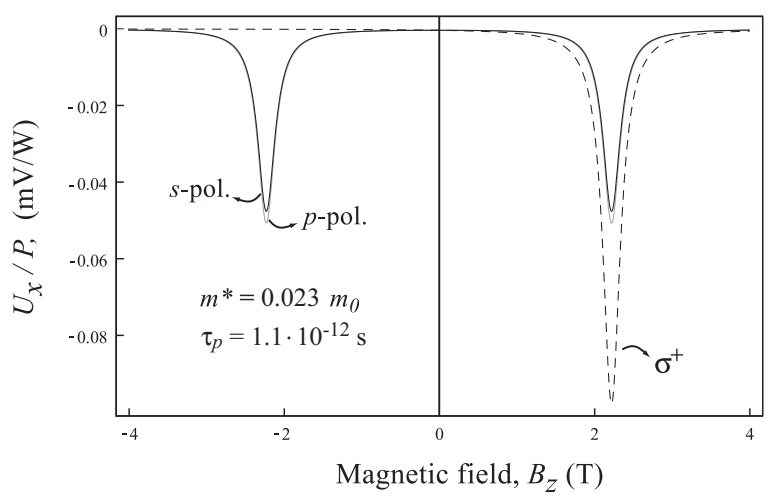

FIG. 8. Theoretical dependencies of $U_{x} / P$ on magnetic field for $s, p$, and $\sigma^{+}$polarizations. In the calculations the transmission coefficients $t_{s}$ and $t_{p}$ are set to unity for simplicity. field dependence of the photocurrent, see Figs. 1 and 2, which does not show any peculiarities at $B=B_{c} / 2$ at which for the opposite unequality an additional resonance should appear, see Eqs. (10).

Finally, the calculations show that the amplitudes of the resonance are nearly the same for $s$ - and $p$-polarized radiation, which agrees well with experimental findings, see Figs. 1 and 2. Indeed, the polarization dependence of the photon drag current is described by $\left|E_{\omega, \|}\right|^{2}$, see Eqs. (11), which for $s$ and $p$ polarizations are given by $\left|E_{\omega,\|,\|}\right|^{2}=2 \pi t_{s}^{2} I_{0} / c$ and $\left|E_{\omega, \|}\right|^{2}=2 \pi t_{p}^{2} I_{0} \cos ^{2} \theta / c$, respectively. Here $t_{s}$ and $t_{p}$ are Fresnel transmission coefficients for $s$ and $p$ waves, $I_{0}$ is the radiation intensity, $\theta$ is the angle of refraction with $\sin \theta=\sin \theta_{0} / n_{\omega}$, and $n_{\omega}$ is the refraction index. Consequently for small angle of incidence with $\cos \theta \approx 1$ and $t_{p} \approx t_{s}$ the fields $\left|E_{\omega, \|}\right|$ and the resulting photocurrents are close to each other for considered polarization states. Figures 7 and 8 also show the photocurrent excited by right-handed, $\sigma^{+}$, circularly polarized radiation. It is seen that, as expected for CR, here the resonant signal is generated for one magnetic field polarity only (positive $B_{z}$ ) and that its magnitude is enhanced by factor 2 compared to that excited by linearly polarized radiation.

Now we briefly address the observed oscillations of the photocurrent. The oscillations have been detected at low temperature and applying cw laser radiation only. They correlate with Shubnikov-de Haas oscillations and like them vanish at high lattice or electron temperature. The latter has been proved applying pulsed high power radiation resulting in a strong electron gas heating which manifest itself in the substantial photoconductive signal [9]. In this particular case even at $T=4.2 \mathrm{~K}$ we only detected the resonant photocurrent. The correlation with $\mathrm{SdH}$ indicates that the resonances are caused by periodic modification of the electron scattering, however the detailed mechanism of the oscillating photocurrent remains unclear and requires further experimental and theoretical studies. In particular, the observed two times difference in the oscillation frequencies should be explored.

\section{SUMMARY}

We have demonstrated that, in InSb/InAlSb QWs, the photon drag effect resulting from the transfer of photon momentum to free electrons is drastically enhanced under cyclotron resonance conditions. The quasiclassical theory of the photon drag effect in two-dimensional electron systems with parabolic energy spectrum developed in the framework of the Boltzmann equation describes well all experimental findings. The observed effect provides a complimentary method to study the details of the band structure and carrier dynamic in InSb-based two-dimensional structures. In particular, almost identical Lorentz profiles and the resonance positions, observed in the experiments on photocurrent excited by low power $\mathrm{THz}$ radiation and the transmission measurement, demonstrate that the photon drag effect can be applied for measuring the carrier effective mass and momentum relaxation time. Due to high sensitivity, this method can be used even under the conditions where the transmission signals are unresolvable, e.g., for structures with a low carrier density or at high temperatures. Furthermore, the investigation of the resonant photocurrent at 
the magnetic field equal to the half of the field corresponding to the cyclotron resonance can provide information on the energy dependence of the momentum relaxation time, which is not easily accessible by other experimental methods.

\section{ACKNOWLEDGMENTS}

We acknowledge financial support from the DFG (SFB 689), the Linkage Grant of IB of BMBF at DLR, RFBR, RF President Grants MD-3098.2014.2 and NSh-1085.2014.2, and "Dynasty" Foundation.
[1] T. Ashley, L. Buckley, S. Datta, M. T. Emeny, D. G. Hayes, K. P. Hilton, R. Jefferies, T. J. Phillips, D. J. Wallis, P. J. Wilding, and R. Chau, Electron. Lett. 43, 14 (2007).

[2] A. M. Gilbertson, A. Kormányos, P. D. Buckle, M. Fearn, T. Ashley, C. J. Lambert, S. A. Solin, and L. F. Cohen, Appl. Phys. Lett. 99, 242101 (2011).

[3] A. M. Gilbertson, M. Fearn, A. Kormányos, D. E. Read, C. J. Lambert, M. T. Emeny, T. Ashley, S. A. Solin, and L. F. Cohen, Phys. Rev. B 83, 075304 (2011)

[4] N. Goel, J. Graham, J. C. Keay, K. Suzuki, S. Miyashita, M. B. Santos, and Y. Hirayama, Physica E 26, 455 (2005).

[5] A. M. Gilbertson, M. Fearn, J. H. Jefferson, B. N. Murdin, P. D. Buckle, and L. F. Cohen, Phys. Rev. B 77, 165335 (2008).

[6] A. M. Gilbertson, W. R. Branford, M. Fearn, L. Buckle, P. D. Buckle, T. Ashley, and L. F. Cohen, Phys. Rev. B 79, 235333 (2009).

[7] N. G. A. Khodaparast, R. C. Meyer, X. H. Zhang, T. Kasturiarachchi, R. E. Doezema, S. J. Chung, N. Goel, M. B. Santos, and Y. J. Wang, Physica E 20, 386 (2004).

[8] A. Rogalski, Infrared Detectors (CRC, Boca Raton, FL, 2010).

[9] S. D. Ganichev and W. Prettl, Intense Terahertz Excitation of Semiconductors (Oxford University Press, Oxford, 2006).

[10] E. Bründermann, H.-W. Hübers, and M. F. Kimmitt, Terahertz Techniques in Springer Series in Optical Sciences (Springer, Berlin, 2012).

[11] M. Frazier, J. A. Waugh, J. J. Heremans, M. B. Santos, X. Liu, and G. A. Khodaparast, J. Appl. Phys. 106, 103513 (2009).

[12] S. Stachel, P. Olbrich, C. Zoth, U. Hagner, T. Stangl, C. Karl, P. Lutz, V. V. Bel'kov, S. K. Clowes, T. Ashley, A. M. Gilbertson, and S. D. Ganichev, Phys. Rev. B 85, 045305 (2012).

[13] K. Seeger, Semiconductor Physics (Springer, Berlin, 1985).

[14] J. M. S. Orr, K.-C. Chuang, R. J. Nicholas, L. Buckle, M. T. Emeny, and P. D. Buckle, Phys. Rev. B 79, 235302 (2009).

[15] G. A. Khodaparast, D. C. Larrabee, J. Kono, D. S. King, S. J. Chung, and M. B. Santos, Phys. Rev. B 67, 035307 (2003).

[16] F. Gouider, Yu. B. Vasilyev, M. Bugár, J. Könemann, P. D. Buckle, and G. Nachtwei, Phys. Rev. B 81, 155304 (2010).

[17] E. Ziemann, S. D. Ganichev, I. N. Yassievich, V. I. Perel, and W. Prettl, J. Appl. Phys. 87, 3843 (2000).

[18] S. D. Ganichev, S. A. Emel'yanov, and I. D. Yaroshetskii, Pis'ma Zh. Èksp. Teor. Fiz. 35, 297 (1982) [JETP Lett. 35, 368 (1982)].

[19] S. D. Ganichev, E. Ziemann, Th. Gleim, W. Prettl, I. N. Yassievich, V. I. Perel, I. Wilke, and E. E. Haller, Phys. Rev. Lett. 80, 2409 (1998).

[20] S. D. Ganichev, Ya. V. Terent'ev, and I. D. Yaroshetskii, Pis'ma Zh. Tekh. Fiz 11, 46 (1985) [Sov. Tech. Phys. Lett. 11, 20 (1985)]
[21] Note that at normal incidence the signal is not detected for any magnetic field strength.

[22] O. J. Pooley, A. M. Gilbertson, P. D. Buckle, R. S. Hall, L. Buckle, M. T. Emeny, M. Fearn, L. F. Cohen, and T. Ashley, New J. Phys. 12, 053022 (2010).

[23] A. M. Gilbertson, P. D. Buckle, M. T. Emeny, T. Ashley, and L. F. Cohen, Phys. Rev. B 84, 075474 (2011).

[24] B. Nedniyom, R. J. Nicholas, M. T. Emeny, L. Buckle, A. M. Gilbertson, P. D. Buckle, and T. Ashley, Phys. Rev. B 80, 125328 (2009).

[25] S. D. Ganichev, E. L. Ivchenko, and W. Prettl, Physica E 14, 166 (2002).

[26] E. L. Ivchenko, Optical Spectroscopy of Semiconductor Nanostructures (Alpha Science, Harrow, UK, 2005).

[27] Note that generally speaking in the presence of magnetic field the photocurrent may also be generated by a magnetogyrotropic photogalvanic effect [28]. However, for the point group $C_{2 v}$ this effect vanishes for $\boldsymbol{B} \| z$ [29] and therefore is disregarded in the further consideration.

[28] V. V. Bel'kov, S. D. Ganichev, E. L. Ivchenko, S. A. Tarasenko, W. Weber, S. Giglberger, M. Olteanu, H.-P. Tranitz, S. N. Danilov, Petra Schneider, W. Wegscheider, D. Weiss, and W. Prettl, J. Phys. C: Condens. Matter 17, 3405 (2005).

[29] V. V. Bel'kov and S. D. Ganichev, Semicond. Sci. Technol. 23, 114003 (2008).

[30] H. M. Barlow, Nature (London) 173, 41 (1954).

[31] V. I. Perel' and Ya. M. Pinskii, Sov. Phys. Solid State 15, 688 (1973).

[32] J. Karch, P. Olbrich, M. Schmalzbauer, C. Zoth, C. Brinsteiner, M. Fehrenbacher, U. Wurstbauer, M. M. Glazov, S. A. Tarasenko, E. L. Ivchenko, D. Weiss, J. Eroms, R. Yakimova, S. Lara-Avila, S. Kubatkin, and S. D. Ganichev, Phys. Rev. Lett. 105, 227402 (2010).

[33] M. M. Glazov and S. D. Ganichev, Phys. Rep. 535, 101 (2014).

[34] Ya. M. Pinskii, Sov. Phys. Solid State 15, 977 (1973).

[35] A. P. Dmitriev, S. A. Emelyanov, S. V. Ivanov, P. S. Kop'ev, Ya. V. Terent'ev, and I. D. Yaroshetsky, Pis'ma Zh. Eksp. Teor. Fiz. 54, 460 (1991) [JETP Lett. 54, 462 (1991)].

[36] B. E. A. Saleh and M. C. Teich, Fundamentals of Photonics (John Wiley and Sons, New York, 2003).

[37] The absence of a noticeable energy dependence of the relaxation time $\tau_{p}$ is additionally supported by the fact that no resonant photocurrent is observed at $\omega=2 \omega_{c}$ which is expected from Eq. (10) otherwise.

[38] Note that while the longitudinal voltage $U_{x}$ is nearly independent of distance between contact and sample edge the transverse one $U_{y}$ is sensitive to the contact positions. 